\title{
Determination of the Singularity-Free Compatible Reachable Workspace for Different Types of Three Degrees-of-Freedom Parallel Manipulators
}

\author{
C. K. Huang, K. Y. Tsai* \\ Department of Mechanical Engineering, National Taiwan University of Science and Technology, Taiwan. \\ * Corresponding author. Tel.: +886-2-2737-6491; email: kytsai@mail.ntust.edu.tw \\ Manuscript submitted January 26, 2016; accepted April 12, 2016. \\ doi: 10.17706/ijcee.2016.8.2.117-131
}

\begin{abstract}
General methods based on inverse kinematics are presented to develop singularity-free compatible workspaces for three degrees-of-freedom parallel manipulators. The characteristics of boundary surfaces are investigated. A workspace boundary surface consists of six or more patches generated by different sets of constraint equations. Each set includes one equation from boundary constraints and several equations from inverse kinematic constraints. The equations for all the patches can be determined by solving direct kinematics once and three times, respectively, for symmetrical and non-symmetrical manipulators. The methods are applicable to existing 3-limb special manipulators with simple inverse kinematics and general 3-limb manipulators without any spherical joints. The effect of passive joint limits, link interactions and singularity are considered in the proposed methods.
\end{abstract}

Key words: 3-DOF, parallel manipulator, compatible, workspace, singularity.

\section{Introduction}

Workspace is one of the most important criteria in the design of parallel manipulators. The reachable workspace is a set of points that can be reached by the tool center point (TCP) on the moving platform. A point is in the workspace if the point can be approached by at least one orientation of the platform. Discretization methods are commonly used to develop the workspace for 6-DOF manipulators. The methods are easy to implement, and they are applicable to different types of parallel manipulators [1]-[3]. However, searching for the exact boundary of the workspace using a discretization method is a very time-consuming task, and the obtained result may be a theoretical workspace, as some of its subspaces might not be reachable through a continuous motion starting from the initial assembly configuration. The exact workspace boundary can be developed using analytical methods, but the process is extremely complicated because the boundary is not a smooth surface. It has been shown the workspace boundary of a 6-DOF parallel manipulator consists of several patches generated by different sets of four constraint equations and efficient methods have been proposed to determine the exact equations for each boundary patch [4]. The existing methods, however, are not applicable to 3-DOF parallel manipulators. This work employs inverse kinematics to develop compatible workspaces (defined as the set of all attainable positions that can be reached through a continuous motion, beginning from the initial assembly configuration) for 
different types of 3-DOF spatial parallel manipulators. The proposed method continuously develops the exact boundary of a compatible workspace. The effects of actuator joint limits, passive joint limits link interactions and singularity are considered in the development process.

Six parameters are required to specify the position and orientation of the platform, so two dimensional boundary patches should be generated by different sets of four boundary constraints [4]. In theory, a boundary patch of a 3-DOF manipulator can be generated by one boundary constraint. Since inverse kinematic solutions cannot be obtained by giving arbitrary six parameters for the position and orientation of the platform, additional kinematic constraints on the parameters are needed so inverse kinematic solutions can be developed if the constraints are satisfied. This work shows three kinematic constraints can be developed for most spatial 3-DOF parallel manipulators with each limb connected to the platform via a spherical joint. Therefore, different boundary patches that can be developed using six variables and four constraint equations: one boundary constraint and three kinematic constraints. For general manipulators or manipulators without any spherical joints, some equations contain additional joint variables so more kinematic constraints are needed to develop a workspace.

This paper first presents constraint equations for developing workspaces for different types of manipulators. Given actuator joint ranges, the proposed methods can develop different types of boundary patches generated by actuator joint limits, passive joint limits, inverse kinematic singularity and link interactions.

A manipulator can switch into a different branch of inverse kinematic solutions at inverse kinematic singular configurations and might not change back to the original branch of solutions [5], [6]. The manipulator cannot balance external wrenches acting on the platform at direct kinematic singular configurations, and link interactions should not occur for any types of manipulator. Therefore, a practical design should have a singularity-free and link-interaction-free workspace. It does not take much time to search for a singularity-free and link-interaction-free joint space. The desired workspace can be developed from the joint space. How to choose proper passive joint ranges is also studied. The results show most boundary surfaces consist of six patches generated by the six actuator joint limits, and it usually takes less than five seconds to develop a workspace when the joint space is given.

\section{Boundary Constraints}

This section develops boundary constraint equations needed to develop the boundary of a workspace. The equations for a general RRS limb and a general UPU limb are developed for illustration. The equations related to other types of limbs with one spherical joint or without any spherical joints can be obtained using similar approaches.

Fig. 1 shows two 3-limb 3-DOF parallel manipulators with related coordinate frames, where $F_{0}, F_{p}$ and $F_{i 1}$ are, respectively, the fixed reference frame, the moving coordinate frame on the platform and the fixed coordinate frame on the base for limb $i$. For the 3-UPU manipulator in Fig. 1(b), an additional frame $F_{i 2}$ is attached on the platform for limb $i$. Let ${ }^{\mathbf{B}} \mathbf{T}_{\mathbf{A}}$ denote the homogeneous matrix that transforms coordinates from frame A to frame $\mathrm{B}$, and the coordinates or position vector of a point $\mathrm{S}$ related to $F_{0}, F_{p}, F_{i 1}$ and $F_{i 2}$ be denoted as $\mathbf{S}, \mathbf{S}^{\prime},{ }^{i 1} \mathbf{S}$ and ${ }^{i 2} \mathbf{S}$ respectively. Then the position vector of $S_{i}$ in Fig. 1(a) related to $F_{0}$ and $F_{p}$ can be determined by

$$
\left[\begin{array}{c}
\mathbf{s}_{i} \\
1
\end{array}\right]={ }^{\mathbf{0}} \mathbf{T}_{\mathbf{p}}\left[\begin{array}{c}
\mathbf{s}_{\boldsymbol{i}}^{\prime} \\
1
\end{array}\right]
$$

and 


$$
\left[\begin{array}{c}
{ }^{i 1} \mathbf{S}_{i} \\
1
\end{array}\right]={ }^{\mathbf{0}} \mathbf{T}_{i \mathbf{1}}^{-1}\left[\begin{array}{c}
\mathbf{S}_{\boldsymbol{i}} \\
1
\end{array}\right]
$$

where $\mathbf{s}_{\boldsymbol{i}}^{\prime}$ is a constant vector, ${ }^{\mathbf{0}} \mathbf{T}_{\boldsymbol{i} \mathbf{1}}$ is a constant matrix, and matrix ${ }^{\mathbf{0}} \mathbf{T}_{\mathbf{p}}$ is defined by the six parameters that specify the coordinates, $(x, y, z)$, of the tool center point (TCP) on the platform and three Euler angles, $(\alpha, \beta, \gamma)$, that define the orientation of the platform. This work uses Z-Y-Z Euler angles to specify the orientation of the platform. The coordinates of $\mathbf{s}_{\boldsymbol{i}}$ and ${ }^{i 1} \mathbf{s}_{\boldsymbol{i}}$ can be expressed as functions of $x, y, z, \alpha, \beta, \gamma$ using the two equations.

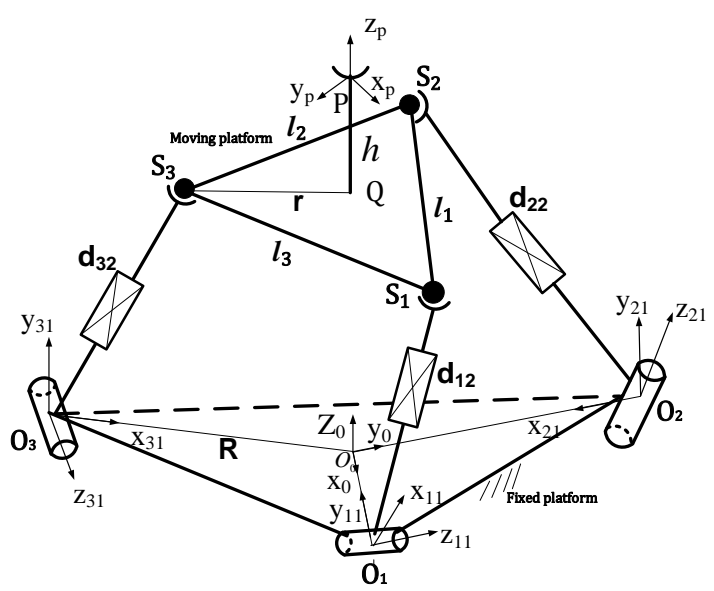

(a) 3-RPS

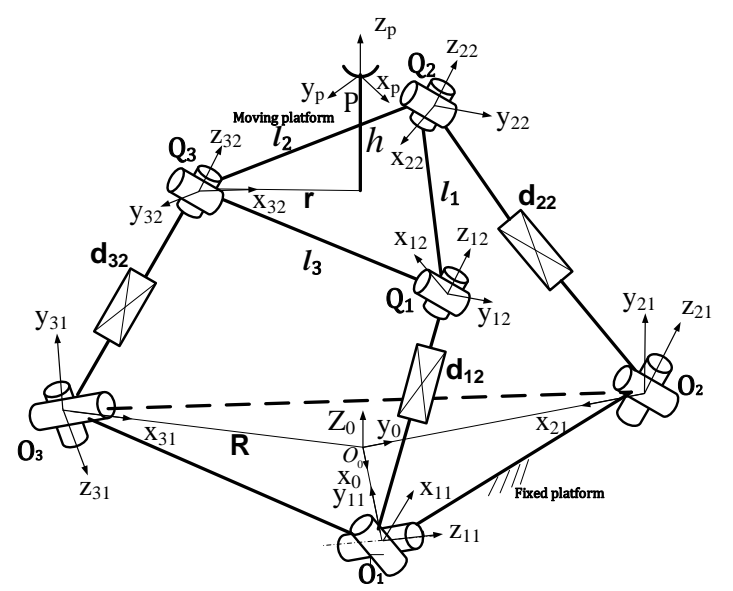

(b) 3-UPU

Fig. 1.3-DOF parallel manipulators.

For an RRS serial chain, the coordinates of ${ }^{\boldsymbol{i}} \mathbf{s}_{\boldsymbol{i}}$ can also be expressed as a functions of the link parameters:

$$
\begin{gathered}
x_{i}=a_{i 2} c \theta_{i 1} c \theta_{i 2}-a_{i 2} c \alpha_{i 1} s \theta_{i 1} s \theta_{i 2}+d_{i 2} s \alpha_{i 1} s \theta_{i 1}+a_{i 1} c \theta_{i 1} \\
y_{i}=a_{i 2} s \theta_{i 1} c \theta_{i 2}+a_{i 2} c \alpha_{i 1} c \theta_{i 1} s \theta_{i 2}-d_{i 2} s \alpha_{i 1} c \theta_{i 1}+a_{i 1} s \theta_{i 1} \\
z_{i}=a_{i 2} s \alpha_{i 1} s \theta_{i 2}+d_{i 2} c \alpha_{i 1}+d_{i 1}
\end{gathered}
$$

where $i$ in subscripts denotes the parameters are related to limb $i$. Solving (3) and (4) to express $s \theta_{i 2}$ and $c \theta_{i 2}$ as functions of $s \theta_{i 1}, c \theta_{i 1}, x_{i}, y_{i}$ and using $\left(s \theta_{i 2}\right)^{2}+\left(c \theta_{i 2}\right)^{2}=1$ to develop the following the equation in $\theta_{i 1}$ :

$$
E_{i 1} s \theta_{i 1}+G_{i 1} c \theta_{i 1}+H_{i 1}=0
$$

where the three coefficients are functions of $x_{i}$ and $y_{i}$. A boundary constraint equation $f_{i}(\mathrm{x}, \mathrm{y}, \mathrm{z}, \alpha, \beta, \gamma)=0$ can be obtained by substituting one actuator joint limit $\left(\theta_{i 1 \max }\right.$ or $\left.\theta_{i 1 \mathrm{~min}}\right)$ into the equation and expressing $x_{i}$ and $y_{i}$ as functions of the six parameters: $x, y, z, \alpha, \beta, \gamma$. A boundary constraint from the passive revolute joint limits can be developed by solving (3) and (4) to express $s \theta_{i 1}$ and $c \theta_{i 1}$ as functions of $s \theta_{i 2}, c \theta_{i 2}, x_{i}$, $y_{i}$ and using $\left(s \theta_{i 1}\right)^{2}+\left(c \theta_{i 1}\right)^{2}=1$ to obtain the equation in $\theta_{i 2}$ :

$$
\mathrm{E}_{i 2} s \theta_{i 2}+\mathrm{G}_{i 2} c \theta_{i 2}+\mathrm{H}_{i 2}=0
$$

Substituting passive joint limit $\theta_{i 2 \max }$ (or $\theta_{i 2 \mathrm{~min}}$ ) into the equation yields the corresponding boundary 
constraint equation in the six parameters. If one joint is fixed at a joint limit, the center point of the spherical joint can rotate about the other joint axis to generate a circle, so the constraint equation cannot be developed by substituting $\theta_{i 2 \max }$ or $\theta_{i 2 \min }$ into (5). For 3-PRS and 3-RPS manipulators, (6) and (7) can be expressed as $\mathrm{E}_{i 1} d_{i 1}+\mathrm{G}_{i 1}=0$ and $\mathrm{E}_{i 2} d_{i 2}+\mathrm{G}_{i 2}=0$ respectively. Related boundary constraints can be developed by substituting prismatic joint limits into the equations.

For the 3-UPU manipulator in Fig. 1(b), the six constraint equations from the actuator joint limits, $d_{i 3 \max }$ and $d_{i 3 \min }$, can be developed by substituting $d_{i 3 \max }=d_{i 3}$ or $d_{i 3 \min }=d_{i 3}$ into following equation:

$$
\left[\mathbf{q}_{i}-\mathbf{o}_{i}\right]^{t}\left[\mathbf{q}_{i}-\mathbf{o}_{i}\right]=d_{i 3}^{2} \text { for } i=1 \text { to } 3
$$

The fourth column of matrix ${ }^{0} \mathbf{T}_{\mathbf{p}}{ }^{\mathbf{p}} \mathbf{T}_{\boldsymbol{i} 2}$ gives $\mathbf{q}_{\boldsymbol{i}}$ with coordinates as functions of the six parameters. The coordinates of ${ }^{\boldsymbol{i}} \mathbf{q}_{\boldsymbol{i}}$ can be expressed as functions of the link parameters:

$$
\begin{gathered}
x_{i}=d_{i 3}\left(c \theta_{i 1} s \theta_{i 2} s \alpha_{i 2}+s \theta_{i 1} c \theta_{i 2} c \alpha_{i 1} s \alpha_{i 2}+s \theta_{i 1} s \alpha_{i 1} c \alpha_{i 2}\right) \\
y_{i}=d_{i 3}\left(s \theta_{i 1} s \theta_{i 2} s \alpha_{i 2}-c \theta_{i 1} c \theta_{i 2} c \alpha_{i 1} s \alpha_{i 2}-c \theta_{i 1} s \alpha_{i 1} c \alpha_{i 2}\right) \\
z_{i}=d_{i 3}\left(-c \theta_{i 2} s \alpha_{i 1} s \alpha_{i 2}+c \alpha_{i 1} c \alpha_{i 2}\right)
\end{gathered}
$$

The boundary constraint for the passive joint limits of $\theta_{i 1}$ and $\theta_{i 2}$ can be obtained using the same method in developing (6) and (7). The equations are similar to (6) and (7) except the six coefficients contain $d_{i 3}$. The constraints in the six parameters can be obtained by substituting $d_{i 3}=\sqrt{\left[\mathbf{q}_{i}-\mathbf{o}_{i}\right]^{t}\left[\mathbf{q}_{i}-\mathbf{o}_{i}\right]}$ into the equations. For the U-joint at point $Q_{i}$, the position vector of point $Q_{i}$ with respect to frame $F_{i 2}$ can be determined by

$$
\left[\begin{array}{c}
{ }^{i 2} \mathbf{o}_{i} \\
1
\end{array}\right]={ }^{\mathbf{p}} \mathbf{T}_{i 2}^{-1}{ }^{\mathbf{0}} \mathbf{T}_{\mathbf{p}}^{-1}\left[\begin{array}{c}
\mathbf{o}_{i} \\
1
\end{array}\right]
$$

where $\mathbf{o}_{\boldsymbol{i}}$ is a constant vector and ${ }^{\mathbf{p}_{\mathbf{i}}} \mathbf{T}_{\boldsymbol{i}}$ is a constant matrix. The coordinates of ${ }^{\boldsymbol{i}} \mathbf{o}_{\boldsymbol{i}}$ are functions of the six parameters. If inverse kinematic solutions exist for a given set of the six parameters (or the six parameters satisfy the inverse kinematic constraints presented in the following section), similar boundary constraints in $\theta_{i 4}$ and $\theta_{i 5}$ for the U-joint at $Q_{i}$ can be developed using the same approach for the U-joint at $O_{i}$. Substituting the joint limits into related equations gives a total of 24 boundary constraint equations from the passive joint limits.

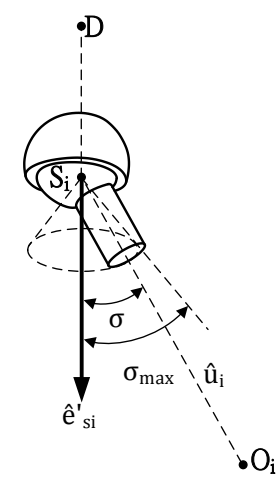

(a) A spherical joint.

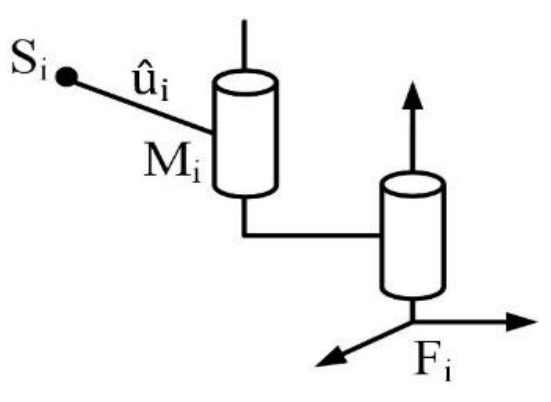

(b) Vector $\widehat{\boldsymbol{u}}_{\boldsymbol{i}}$ for a general limb.

Fig. 2. Mechanical limit for a spherical joint. 
Fig. 2 shows the mechanical limit for a spherical joint on the platform, where unit vector $\widehat{\boldsymbol{u}}_{\boldsymbol{i}}$ represents the direction of the center line of the link and unit vector $\hat{\mathbf{e}}_{\boldsymbol{s} i}^{\prime}$ that defines the axis of symmetry of the spherical joint is a constant vector with respect to the moving frame on the platform. Let ${ }^{\mathbf{0}} \mathbf{R}_{\mathbf{p}}$ denote the rotation matrix of ${ }^{0} \mathbf{T}_{\mathbf{p}}$. Then $\hat{\mathbf{e}}_{\boldsymbol{s i}}={ }^{\mathbf{0}} \mathbf{R}_{\mathbf{p}} \hat{\mathbf{e}}^{\prime}{ }_{\boldsymbol{s i}}$ with the three components as functions of the six parameters. The boundary constraint equation from the passive spherical joint limit can be expressed as

$$
\hat{\mathbf{e}}_{\boldsymbol{s i}} \cdot \widehat{\boldsymbol{u}}_{\boldsymbol{i}}=\cos \sigma_{\max }
$$

where $\sigma_{\max }$ denotes the maximum allowable angle of the joint. Vector $\widehat{\boldsymbol{u}}_{\boldsymbol{i}}$ for the general limb in Fig. 2(b) can be determined by

$$
\widehat{\boldsymbol{u}}_{i}=\frac{\mathbf{m}_{i}-\mathbf{s}_{i}}{\left\|\mathbf{m}_{i}-\mathbf{s}_{i}\right\|} \text { with }\left[\begin{array}{c}
\mathbf{m}_{i} \\
\mathbf{1}
\end{array}\right]={ }^{0} \mathbf{T}_{i 1} \mathbf{A}_{i 1}\left[\begin{array}{c}
0 \\
\mathbf{0} \\
d_{i 2} \\
1
\end{array}\right]
$$

where $\mathbf{A}_{\boldsymbol{i 1}}$ denotes the $\mathrm{D}-\mathrm{H}$ transformation matrix $\mathbf{A}_{\mathbf{1}}$ for limb $i$. The vector developed using $\mathbf{A}_{\boldsymbol{i 1}}$, however, includes the first joint variable of limb $i$. For special 3-RPS in Fig. 1(a) or 3-US parallel manipulators, the coordinates for the origin $\mathrm{O}_{i}$ of frame $F_{i 1}$ are constants with respective to frame $F_{0}$, so unit vector $\hat{\boldsymbol{u}}_{i} \equiv\left(\mathbf{o}_{i}-\mathbf{s}_{i}\right) /\left\|\mathbf{o}_{i}-\mathbf{s}_{i}\right\|$ can be developed without the first joint variable of limb $i$. The vector norm $\left\|\mathbf{o}_{i}-\mathbf{s}_{i}\right\|$ is a constant for 3-US parallel manipulators. The coordinates of reference point $M_{i}$ to develop $\widehat{\boldsymbol{u}}_{\boldsymbol{i}}$ for the special 3-CS manipulator in Fig. 3 can be determined by

$$
\mathbf{m}_{i}=\mathbf{o}_{i}+d_{i 1} \mathbf{z}_{i 1}
$$

where the coordinates of the origin $\mathrm{O}_{i}$ and the direction of $\mathbf{z}_{\mathbf{i 1}}$ are constants and $d_{i 1}$ is the $\mathrm{z}$ coordinate of ${ }^{\boldsymbol{i} 1} \mathbf{s}_{\boldsymbol{i}}$ which is a function of the six parameters. Therefore, the unit vector defined by $\hat{\boldsymbol{u}}_{\boldsymbol{i}} \equiv\left(\mathbf{q}_{i}-\mathbf{s}_{i}\right) /\left\|\mathbf{q}_{i}-\mathbf{s}_{i}\right\|$ is independent of the first joint variable. More equations are needed to develop a workspace if the boundary constraints have additional joint variables (besides the six parameters for the position and orientation of the platform). Link interactions rarely occur for 3-limb parallel manipulators. The equation for link interactions in the previous work is used as the boundary constraint to develop related patches if they exist [7].

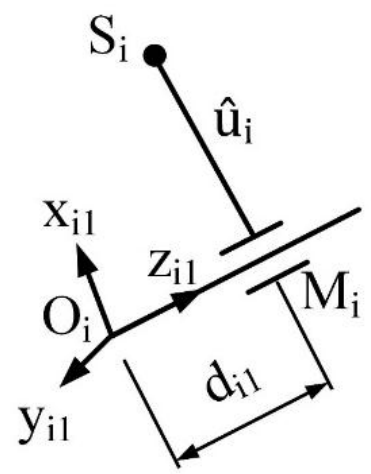

Fig. 3. Vector $\widehat{\boldsymbol{u}}_{\boldsymbol{i}}$ for a special 3-CS manipulator. 


\section{Kinematic Constraints and Singularity-Free Joint Space}

Inverse kinematic solutions cannot be developed for 3-DOF manipulators unless some kinematic constraints on the six parameters are satisfied. For manipulators with three spherical joints on the platform, the solutions exist if point $S_{i}$ on the surface generated by the $i^{\text {th }}$ limb for $i=1,2,3$. The surface generated by point $S_{i}$ on the $i^{\text {th }}$ limb of a 3-RRS manipulator can be developed from (5) and (7). First, express $s \theta_{i 2}$ in (5) as a function of $\mathrm{z}_{i}$ and the link parameters in the equation. Next, substitute the function into (7) so $c \theta_{i 2}$ can also be expressed as a function of $z_{i}, \mathrm{E}_{i 2}, \mathrm{G}_{i 2}, \mathrm{H}_{i 2}$ and the link parameters. The equation for the surface can be obtained using $\left(s \theta_{i 2}\right)^{2}+\left(c \theta_{i 2}\right)^{2}=1$. The equations in local coordinates (related to local coordinate frame $F_{i 1}$ ) for the surfaces of the general RRS, RPS, PRS and PPS limbs are given in Table 1. For a 3-limb parallel manipulator, three kinematic constraints can be developed using (2) to express $x_{i}, y_{i}, z_{i}$ as functions of the six parameters.

Loop equations can be used as the kinematic constraint equations, but the equations involve too many joint variables, so more equations are needed to develop a workspace. For the five axes of the $i^{\text {th }}$ limb of a 3-UPU manipulator shown in Fig. 4, the direction of the second axis, $\widehat{\boldsymbol{u}}_{i 2}$, is a function of the first joint displacement, $\theta_{i 1}$, and the direction of the fourth axis, $\widehat{\boldsymbol{u}}_{i 4}$, is a function of the fifth joint displacement, $\theta_{i 5}$, and the six parameters. Inverse kinematic solutions of a 3-UPU manipulator exist if the following three kinematic constraints are satisfied:

$$
\begin{aligned}
& \widehat{\boldsymbol{u}}_{\boldsymbol{i 2}} \cdot \widehat{\boldsymbol{u}}_{\boldsymbol{i} \mathbf{3}}=c_{i 1} \\
& \widehat{\boldsymbol{u}}_{\boldsymbol{i} \mathbf{4}} \cdot \widehat{\boldsymbol{u}}_{\boldsymbol{i} \mathbf{3}}=c_{i 2} \\
& \widehat{\boldsymbol{u}}_{\boldsymbol{i} \mathbf{2}} \cdot \widehat{\boldsymbol{u}}_{\boldsymbol{i} \mathbf{4}}=c_{i 3}
\end{aligned}
$$

\begin{tabular}{|c|c|}
\hline Type & Kinematic constraint \\
\hline RRS & 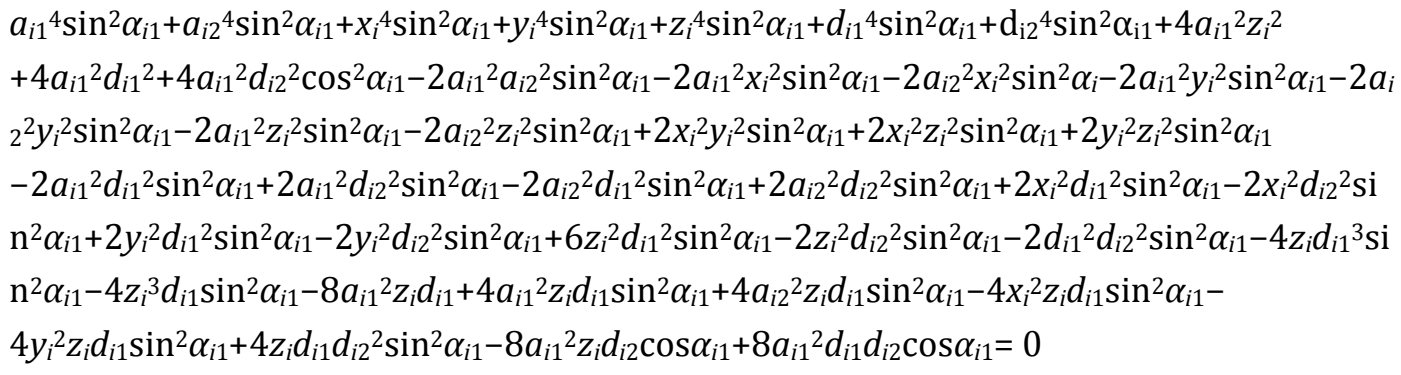 \\
\hline RPS & $\begin{array}{l}a_{i 1}{ }^{2}+a_{i 2}{ }^{2}-x_{i}{ }^{2}-y_{i}^{2}-z_{i}^{2}+d_{i 1}{ }^{2}-2 d_{i 1}\left(d_{i 1}-z_{i}+a_{i 2} \sin \alpha_{i 1} \sin \theta_{i 2}\right)+\left(d_{i 1}-z_{i}+a_{i 2} \sin \alpha_{i 1} \sin \theta_{i 2}\right)^{2} / \cos ^{2} \alpha_{i 1} \\
+2 a_{i 1} a_{i 2} \cos \theta_{i 2}+2 a_{i 2} d_{i 1} \sin \alpha_{i 1} \sin \theta_{i 2}=0\end{array}$ \\
\hline PRS & $\begin{array}{l}a_{i 2}{ }^{2} x_{i} \cos \alpha_{i 1} \cos \theta_{i 1}+a_{i 2}{ }^{2} y_{i} \cos \alpha_{i 1} \sin \theta_{i 1}-a_{i 2}{ }^{2} x_{i} \cos \alpha_{i 1} \cos ^{3} \theta_{i 1}-a_{i 2}^{2} y_{i} \cos \alpha_{i 1} \sin ^{3} \theta_{i 1}-a_{i 2}{ }^{2} x_{i} \cos \alpha_{i 1} \cos \theta_{i} \\
\sin ^{2} \theta_{i 1}-a_{i 2} y_{i} y_{i} \cos \alpha_{i 1} \cos ^{2} \theta_{i 1} \sin \theta_{i 1}=0\end{array}$ \\
\hline PPS & $a_{i 1}+a_{i 2} \cos \theta_{i 2}-x_{i} \cos \theta_{i 1}-y_{i} \sin \theta_{i 1}=0$ \\
\hline
\end{tabular}

Table 1. Kinematic Constraints for Different Limbs

where $c_{i 1}, c_{i 2}, c_{i 3}$ are constants, and unit vector $\widehat{\boldsymbol{u}}_{i 3} \equiv \frac{\mathbf{q}_{i}-\mathbf{o}_{\boldsymbol{i}}}{\left\|\mathbf{q}_{i}-\mathbf{o}_{i}\right\|}$ defines the direction of the actuated prismatic joint. The three limbs give nine kinematic constraint equations in twelve variables: the six parameters for the position and orientation of the platform and six passive joint variables, $\theta_{i 1}$ and $\theta_{i 5}$ for $i=1,2,3$. 


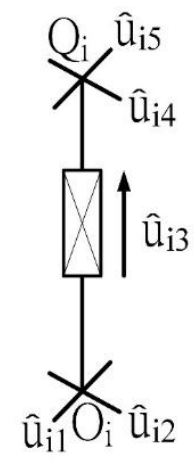

Fig. 4. The five axes of the $i^{\text {th }}$ limb of a 3-UPU manipulator.

Inverse kinematic singularity can generate some boundary patches. Inverse kinematic singularity occurs when the determinant of the Jacobian matrix, $\operatorname{det}(\mathbf{J})$, of one limb is zero. Since the determinant of a 2-DOF serial chain is independent of the first joint displacement, a few second joint displacements for singularity can be determined from the equation $\operatorname{det}(\mathbf{J})=0$ for manipulators with three spherical joints. In theory, related equations for boundary constraints can be obtained by substituting the displacements into (7), but this work uses the displacements to search for a singularity-free joint space. For example, if limb $i$ of a 3-RRS manipulator reaches an inverse kinematic singular configuration at $\theta_{i 2}=\theta_{i \mathrm{~A}}$ or $\theta_{i 2}=\theta_{i \mathrm{~B}}$ with $\theta_{i \mathrm{~B}}>\theta_{i \mathrm{~A}}$, then a (inverse kinematic) singularity-free joint space for limb $i$ can be obtained by letting $\theta_{i \mathrm{~A}}<\theta_{i 2}<\theta_{i \mathrm{~B}}$ for $i$ $=1,2,3$.

There exists a screw that is reciprocal to all the screws of limb $i$ if the limb is not at an inverse kinematic singular configuration. A manipulator reaches a direct singular configuration if the three reciprocal screws from the three limbs are dependent. Let $\$_{i r}$ be the unit reciprocal screw for limb $i$ and $\mathbf{H}$ denote the $6 \times 3$ matrix with $\$_{\text {ir }}$ (for $i=1,2,3$ ) as the column vectors. Then the closeness to direct kinematic singularity can be evaluated by

$$
\mu=\left|\operatorname{det}\left(\mathbf{H}^{\mathbf{t}} \mathbf{H}\right)\right|
$$

This work uses a discretization method to search for a singularity-free joint space. The proposed method also determines the maximum and minimum joint displacements of each passive joint and checks if link interactions occur in the grid-scanning process. For manipulators with three spherical joints, the direct kinematic equations are given below:

$$
\begin{aligned}
& \left(s_{1}-s_{2}\right) \cdot\left(s_{1}-s_{2}\right)=l_{1}^{2} \\
& \left(s_{2}-s_{3}\right) \cdot\left(s_{2}-s_{3}\right)=l_{2}^{2} \\
& \left(s_{3}-s_{1}\right) \cdot\left(s_{3}-s_{1}\right)=l_{3}^{2}
\end{aligned}
$$

where constants $l_{1}, l_{2}$ and $l_{3}$ defined in Fig. 1 (a) are the side lengths of the platform and $\mathbf{s}_{\mathbf{1}}, \mathbf{s}_{\mathbf{2}}$ and $\mathbf{s}_{\mathbf{3}}$ are the functions of joint variables that can be developed using (3)-(5). In the search process, smaller initial ranges of $\theta_{i 1}$ for $i=1,2,3$ are first given. Then, direct kinematics and the scanning method are employed for computing passive joint displacements (and saving maximum and minimum displacements), evaluating the closeness to singularity and checking link interactions. Link interactions are detected using existing equations [7]. If all the grids are free of singularity and link interactions, then increase the size of the joint 
space, scan the extra grids and update the maximum and minimum passive joint displacements. Repeat the process until real kinematic solutions cannot be obtained, the manipulator reaches singular configurations or link interactions occur for some grids. For 3-UPU manipulators, it needs to solve 12 loop equations for direct kinematic solutions and uses the determinant of the Jacobian matrix of each limb to evaluate the closeness to inverse kinematic singularity. If passive joint limits are chosen in a way that the joint ranges include the obtained maximum and minimum passive joint displacements (determined in the grid-scanning process), then the passive joint limits cannot be reached so actuator joint limits will generate all the singularity-free and link-interaction-free boundary patches.

\section{Boundary Surfaces and Algorithms}

This section presents methods to determine the equations for each boundary patch, studies the characteristics of the boundary surfaces and then proposes a general algorithm to develop compatible workspaces for different types of 3-DOF parallel manipulators.

Fig. 5(a) shows the top view of three neighboring boundary patches around the extreme point where the three actuator joints are at their upper joint limits. The number $i$ on the patch indicates the patch is generated by the $i^{\text {th }}$ actuated joint at its upper joint limit. A bifurcation curve is the intersection of two neighboring patches so the union of the numbers of the two neighboring patches gives the two boundary constraints for the curve. On the other hand, the intersection number of two neighboring bifurcation curves determines the boundary constraint that generates the boundary patch in between. The workspace boundary in this work is represented by boundary curves on consecutive cross-sections through extreme point $E_{U}=(1,2,3)$ with the numbers in the parenthesis indicating the actuators on limbs 1,2 and 3 are at their upper limits. The boundary curve on a cross-section of a workspace consists of two or more segments, as shown in Fig. 5(b) where number $j^{\prime}$ denotes the $j^{\text {th }}$ actuated joint is at its lower joint limit. The boundary constraints for the first boundary segments starting from point $\mathrm{E}_{U}$ of the boundary curves on all the cross-sections of a workspace can be predicted by solving the direct kinematics at three specified points in the joint space.

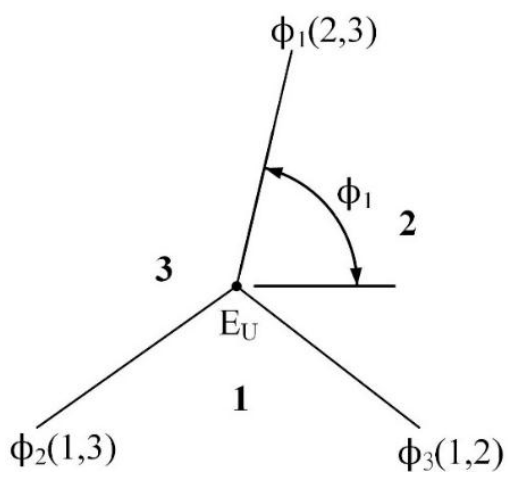

(a) Top view

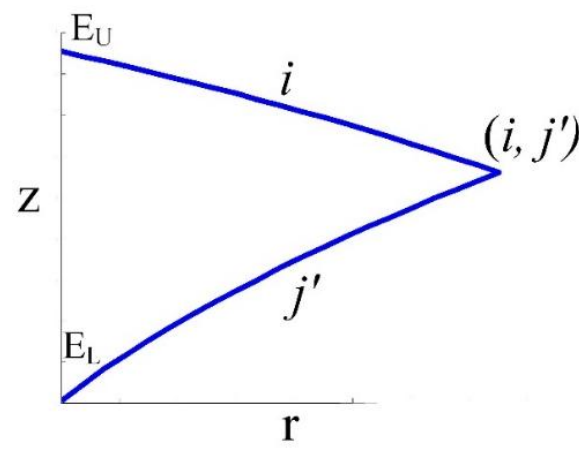

(b) A cross-section

Fig. 5. Boundary surface of a 3-DOF manipulator.

Let $\rho_{i \max }$ and $\rho_{i \min }$ respectively denote the upper joint limit and the lower joint limit of the $i^{\text {th }}$ actuated joint. Then the angle $\phi_{i}$ for the tangent vector of the $i^{\text {th }}$ bifurcation curve can be determined by the $x$ and $y$ coordinates of point $\mathrm{E}_{\mathrm{U}}$ and the next point on the curve obtained by solving the direct kinematics with $\rho_{i}=$ $\rho_{\text {imax }}-\delta$ and the other two limbs at their upper joint limits. The intersection of two sets of numbers from two neighboring bifurcation curves $(2,3)$ related to $\phi_{1}$ and $(3,1)$ related to $\phi_{2}$ determines $\rho_{3 \max }$ generates the region from $\phi_{1}$ to $\phi_{2}$. Likewise, $\rho_{1 \max }$ and $\rho_{2 \max }$ generate the regions from $\phi_{2}$ to $\phi_{3}$ and $\phi_{3}$ to $\phi_{1}$, respectively. For a symmetrical manipulator, $\phi_{2}=\phi_{1}+120^{\circ}$ and $\phi_{3}=\phi_{1}+240^{\circ}$. The angle $\phi$ that defines a 
cross-section is used to determine the boundary constraint for developing the first boundary segment. The boundary curve on the cross-section can be developed using the following equation that transforms the coordinates, $(x, y, z)$, of the TCP on the moving platform into cylindrical coordinates, $\mathrm{r}, \phi$ and $\mathrm{t}$ :

$$
\left(\begin{array}{l}
x \\
y \\
z
\end{array}\right)=\left(\begin{array}{c}
r \cos \eta \\
r \sin \eta \\
0
\end{array}\right)+\left(\begin{array}{l}
0 \\
0 \\
1
\end{array}\right)
$$

With angle $\phi$ that defines the cross-section given, the boundary curve on the cross-section can be developed by changing $r$ and solving for $t$ (and the remaining variables). The boundary constraint for the first segment is used to develop the curve until another constraint $j$ exceeds its joint limits (at the point just passes a bifurcation point). Then use the new constraint to develop the next segment and repeat the process until the path reaches the extreme point $E_{L}=\left(1^{\prime}, 2^{\prime}, 3^{\prime}\right)$ with the three actuators at their lower joint limits. If the solutions cannot be obtained in the forward direction, then develop the path in the backward direction.

For a nonsymmetrical manipulator, the extreme point $E_{L}$ is not on the z-axis of the reference frame. It can solve for $E_{L}$ and then define a new coordinate frame with the straight line through the two extreme points as the new z-axis so a boundary curve can be developed from one extreme point to the other extreme point. The boundary and kinematic constraints can be transformed into equations with $r, \phi$ and $t$ using the following steps: (i) expressing the coordinates of the TCP with respect to the new coordinate frame as functions of $r, \phi$ and $t$ (using (23)); (ii) using the constant transformation matrix that transforms the coordinates from the new frame to $F_{0}$ to express coordinates $x, y$ and $z$ as functions of $r, \phi$ and $t$; and (iii) substituting $x, y$ and $z$ into related constraint equations. If there is no joint variable in the boundary and kinematic constraints, then a boundary patch can be generated by four equations (one from the boundary constraint and three from the kinematic constraints) in the six parameters. The boundary constraint from the spherical joint limit of limb $i$ of a general 3-RRS manipulator has an additional joint variable $\theta_{i 1}$, so one more kinematic constraint with $\theta_{i 1}$ (such as (6)) is needed to develop the patch for the joint limit. For a 3-UPU manipulator, it needs to solve ten equations (one from the boundary constraints and nine from the kinematic constraints) in twelve variables to develop a boundary patch. The steps to develop the workspace of a 3-DOF parallel manipulator are summarized below:

1) Input the link parameters and define related coordinate frames. Search for a singularity-free and link-interaction-free joint space and determine the actuator joint limits $\left(\rho_{i \max }\right.$ and $\rho_{i \min }($ for $i=1,2,3)$ and the passive joint limits.

2) Input increments $\Delta r$ and $\Delta \phi$. Let $\mathrm{r}=\mathrm{s}=0$ and $\rho_{3 \min }$ be the boundary constraint. Let $s=1$ if the manipulator is symmetric.

3) If $s=1$ then (i) solve the direct kinematics to determine $E_{U}$ and $\phi_{1}$; (ii) Let $\phi=\phi_{1}$, and $\phi_{e}=\phi_{1}+120^{\circ}$; (iii) Go to Step 5.

4) Solve the direct kinematics for $E_{U}, E_{L}$ and use them to define the new coordinate frame. Compute $\phi_{1}, \phi_{2}$ and $\phi_{3}$ (related to the new frame). Let $\phi=\phi_{1}$ develop the curve starting from point $E_{U}$.

5) Repeat (i) let $r=r+\Delta r$; (ii) use the boundary constraint to develop the next boundary point; (iii) plot boundary points $(\phi, r, t)$; (iv) plot $\left(\phi+120^{\circ}, r, t\right)$ and $\left(\phi+240^{\circ}, r, t\right)$ if $s=1$; (v) solve the inverse kinematics for the boundary point $(\phi, r, t)$, until a bifurcation point or the extreme point $E_{L}$ is reached. Go to Step 7 if point $E_{L}$ is reached.

6) Let $r=r+\Delta r$ and use the new boundary constraint at the bifurcation point to develop the next boundary point. If the point cannot be obtained, then let $\Delta r=-\Delta r$ and develop the curve in the opposite direction. Go to Step 5. 
7) Let $\phi=\phi+\Delta \phi$. Exit if $s=1$ and $\phi \geq \phi_{e}$. Exit if $\phi \geq \phi+360^{\circ}$. If $\phi>\phi_{2}$, then let $\rho_{1 \max }$ be the boundary constraint. If $\phi>\phi_{3}$, then let $\rho_{2 \max }$ be the boundary constraint. Let $r=0, \Delta r=\mid \Delta r$ land develop the new curve (on the new cross-section) starting from point $E_{U}$. Go to Step 5.

In general, a boundary surface consists of six patches generated by the six actuator joint limits if the passive joint limits cannot be reached. Fig. 1(a) shows a typical boundary curve on a cross-section with one segment generated by an upper limit and the other by a lower limit. Since the lower limit $j$ ' always moves the TCP back to the lower extreme point $\mathrm{E}_{\mathrm{L}}$, this work uses the lower limit $j^{\prime}$ to develop the curve in the opposite direction (instead of the forward direction) when the path reaches the bifurcation point $(i, j)$. Fig. 1(b) shows a special case of boundary curves. If the path is developed in the forward direction from point $\mathrm{A}$, the solutions cannot be obtained for the next point with $r=r+\Delta r$. It needs to develop the next point $\mathrm{B}$ in the opposite direction (with $r=r-\Delta r$ ). To avoid getting point A instead of point $\mathrm{B}$, the proposed method lets $t=$ $t-\Delta t$ (or $t=t+\Delta t$ if the curve is moving upward) and solves for $\mathrm{r}$ to develop point B. Then let $\Delta r=-\Delta r$ and develop the curve in the opposite direction.

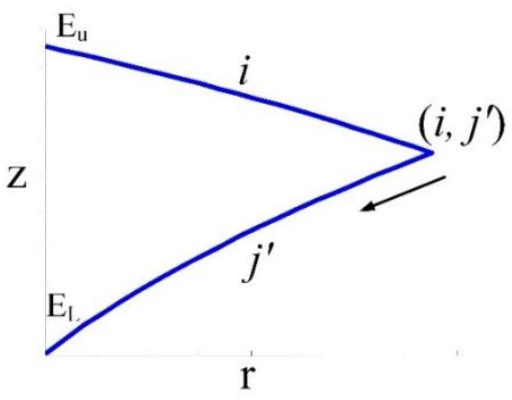

(a) A boundary curve

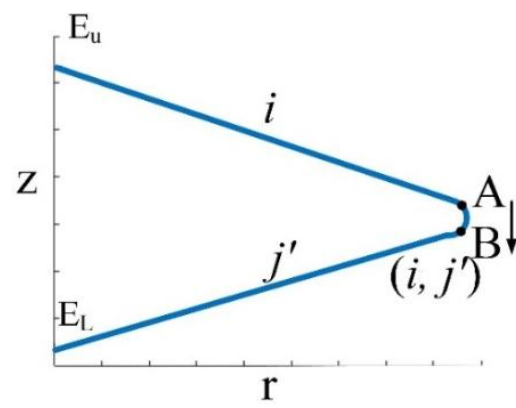

(b) A special boundary curve

Fig. 1. Boundary curves on a cross-section.

\section{Numerical Examples}

The workspaces for three different types of manipulators are studied in this section. Singularity-free and link-interaction-free joint spaces are first determined and then used to develop corresponding workspaces. The time required for developing a joint space and the related workspace using different types of constraints and different numbers of equations is evaluated and compared.

\subsection{3-RPS Manipulator}

For the symmetrical 3-RPS manipulator shown in Fig. 1 (a) with $a_{j 1}=a_{j 2}=0, d_{j 1}=0, \alpha_{j 1}=90^{\circ}$ and $\theta_{j 2}=\alpha_{j 2}=0$, for $j=1,2$, 3. Local coordinates $\mathbf{s}_{\mathbf{1}}^{\prime}=[50,0,-30]^{\mathrm{t}}$ with centimeters as the unit and

$$
F_{11}=\left[\begin{array}{cccc}
-1 & 0 & 0 & 75 \\
0 & -0.2588 & 0.9656 & 0 \\
0 & 0.9656 & 0.2588 & 0 \\
0 & 0 & 0 & 1
\end{array}\right]
$$

Points $\mathbf{s}_{\mathbf{2}}^{\prime}$ and $\mathbf{s}_{\mathbf{3}}^{\prime}$ are respectively determined by rotating $\mathbf{s}_{\mathbf{1}}^{\prime}$ about the $z_{p}$ axis (on the platform) by $120^{\circ}$ and $240^{\circ}$. Frames $F_{21}$ and $F_{31}$ are defined using the same method. All the links are represented by cylinders with a diameter of $3 \mathrm{~cm}$. A RPS limb reaches its singular configuration at $d_{2}=0$. Since the measure in (19) is not invariant to physical units, this work first evaluates the measure at ten disjointed points to obtain the average value $\mu_{\text {ave }}$ and let $\varepsilon=0.05 \mu_{\text {ave }}$ to detect direct kinematic singularity. The search for the joint space starts from $d_{i 2}=40$ for $i=1,2,3$ and exits the search process if the value of $\mu$ at a grid is less than $\varepsilon$ or link interactions occur. Based on the obtained data, the actuator joint ranges $35 \leq d_{i 2} \leq 50$ for $i=1,2,3$ are used 
to develop the workspace. Next, the passive joint ranges are chosen to be $120^{\circ} \leq \theta_{i 1} \leq 160^{\circ}$ and $0^{\circ} \leq \sigma_{i} \leq 30^{\circ}$ (for $i=1,2,3$ ) because the maximum and minimum passive revolute joint displacements and the maximum spherical joint displacement are about $151^{\circ}, 124^{\circ}$ and $15^{\circ}$, respectively. In this case, passive joint limits cannot generate any boundary patches.

Solving direct kinematics at points with $d_{12}=d_{22}=d_{32}=50$ and $d_{22}=d_{32}=50, d_{12}=49.8$ gives extreme point $E_{U}=(0,0,56.1)$ and the coordinates of its neighboring bifurcation point $(0.89,0.08,71.04)$, so $\phi_{1} \approx$ $\operatorname{atan} 2(0.08 / 0.09)=5.6011^{\circ}$ and the boundary constraint developed by substituting $d_{32}=50$ into equation $\mathrm{E}_{32} d_{32}+\mathrm{G}_{32}=0$ (defined in Section 2) will generate the first segments for the cross-sections from $\phi_{1}$ to $\phi_{1}+120^{\circ}$. The kinematic constraints equations can be developed directly from the general equations in Table 1. For this special design, point $S_{i}$ must be on the X-Y pane of frame $F_{i 1}$, so its $z$ coordinate related to $F_{i 1},{ }^{i 1} S_{i z}$, should be zero for $i=1,2,3$. The condition gives three kinematic constraint equations

$$
{ }^{i 1} S_{i z}=0 \text { for } \mathrm{i}=1,2,3
$$

for the inverse kinematics of the 3-RPS parallel manipulator. The boundary surface can be developed by solving four constraint equations in the six parameters that define the position and orientation of the platform.

The workspace given in Fig. 7 is developed from $\phi=10^{\circ}$ to $\phi=120^{\circ}$ with a step of $\Delta \phi=5^{\circ}$. The workspace boundary consists of six patches generated by the six actuator joint limits. If the workspace is developed from the cross-section at $\phi=5.6011^{\circ}$, the first segment might coincide (or partially coincident) with the bifurcation curve from extreme point $E_{U}$ to the extreme point with $(1 ', 2,3)$, or the bifurcation curve might intersect the cross-section at one or more points. The development process is more complicated in this case, so the first cross-section starts from $\phi=10^{\circ}$.

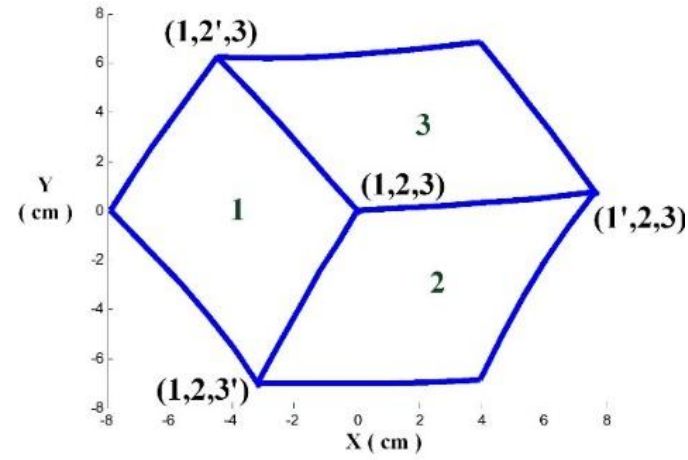

(a) Upper boundary

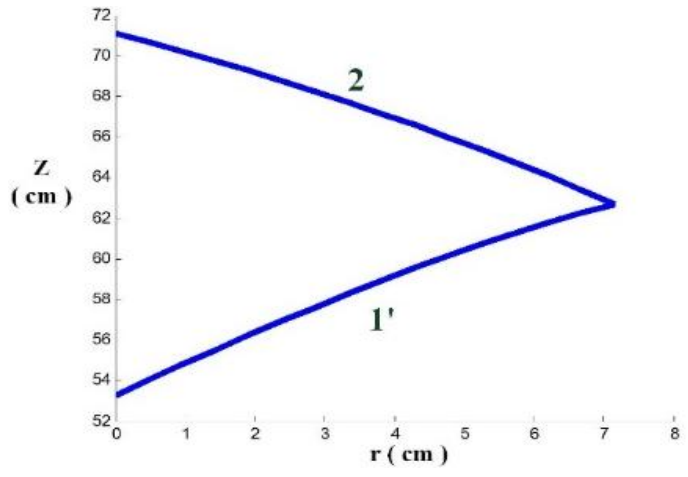

(c) Cross section at $\phi=0$

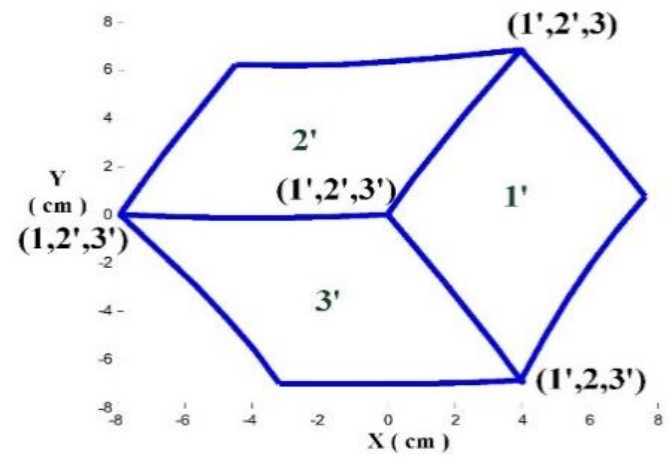

(b) Lower boundary

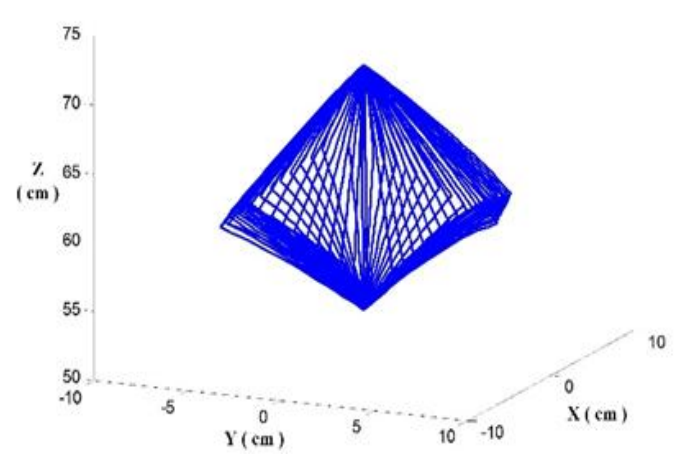

(d) Isometric view

Fig. 7. Workspace of a 3-RPS parallel manipulator. 
Since the cross-sections stay away from the bifurcation curves from $E_{U}$ to its three neighboring extreme points, those curves (including the curves from $E_{L}$ to its three neighboring extreme points) cannot be developed using the proposed algorithm. The curves in Fig. 7(a) and Fig. 7(b) are developed using direct kinematics by fixing two joints in limited positions and moving one joint from one limit to the other limit. The workspace is redeveloped using new spherical joint ranges: $0^{\circ} \leq \sigma_{i} \leq 10^{\circ}$ for $i=1,2,3$. In this case, the three spherical joint limits can generate boundary patches, as shown in Fig. 8, where $S P_{i}$ indicates the patch is generated by the spherical joint limit of limb $i$.

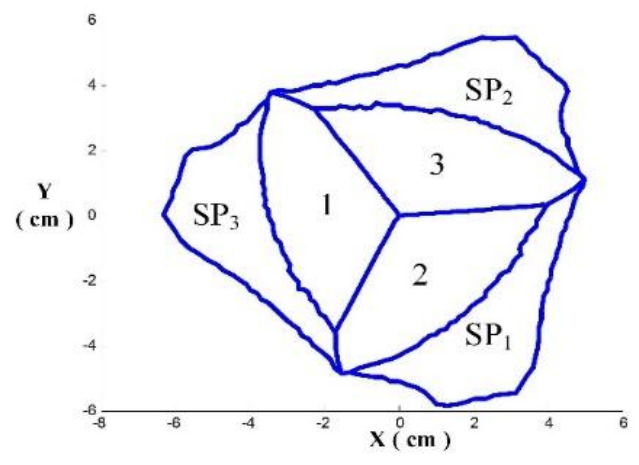

(a) Upper boundary

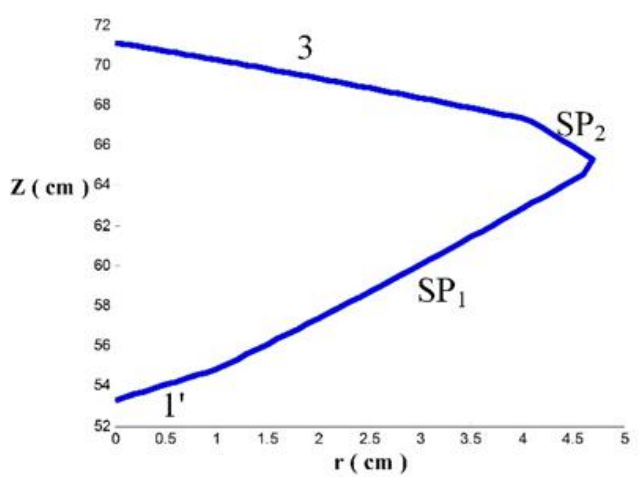

(c) Cross section at $\phi=0^{\circ}$

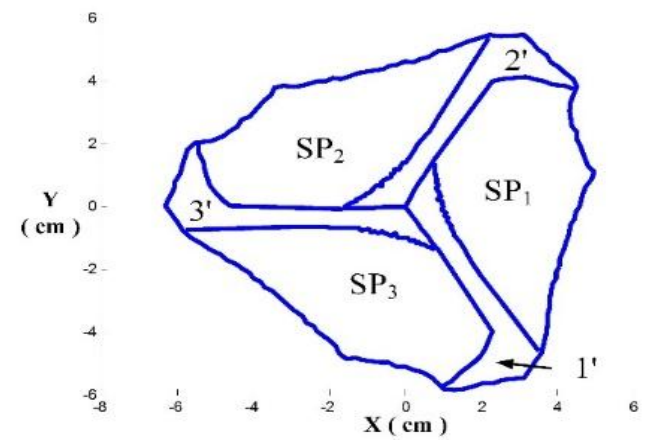

(b) Lower boundary

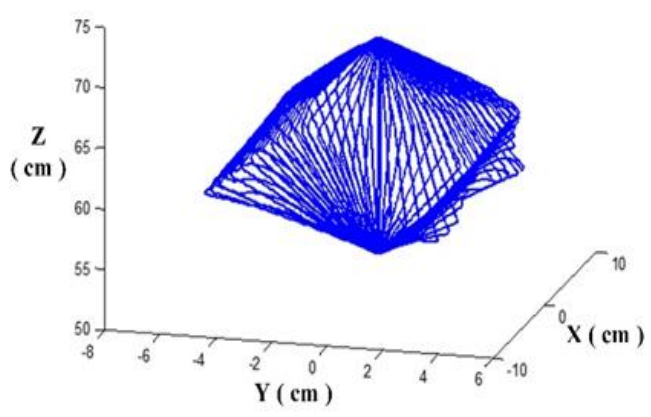

(d) Isometric view

Fig. 8. Workspace of a 3-RPS parallel manipulator with spherical joint limits.

\subsection{3-UPU Manipulator}

All the link lengths $a_{j k}$ (for $j=1,2,3$ and $k=1,2, \cdots, 5$ ) and offsets are zeroes except the actuator displacements $d_{j 3}$. The fixed joint displacement $\theta_{j 3}=10^{\circ}$ and the twisted angles are $\alpha_{j 1}=\alpha_{j 3}=90^{\circ}, \alpha_{j 2}=\alpha_{j 4}=$ $-90^{\circ}, \alpha_{j 5}=0$. The two reference frames for limb 1 are defined by matrices

$$
F_{11}=\left[\begin{array}{cccc}
0.9848 & 0 & 0.1376 & 75 \\
-0.1677 & 0.2588 & 0.9513 & 0 \\
-0.0449 & -0.9659 & 0.2549 & 0 \\
0 & 0 & 0 & 1
\end{array}\right]
$$

and

$$
F_{12}=\left[\begin{array}{cccc}
0.9962 & 0 & 0.0872 & 50 \\
-0.0858 & 0.1736 & 0.9811 & 0 \\
-0.0151 & -0.9848 & 0.1730 & -15 \\
0 & 0 & 0 & 1
\end{array}\right]
$$


The related frames on limbs 2 and 3 are determined by rotating the frames about the respective $\mathrm{z}$-axis by $120^{\circ}$ and $240^{\circ}$. The search process for the desired joint space gives $30 \leq d_{i 3} \leq 41$ for the actuators, and $25^{\circ} \leq$ $\theta_{i 1} \leq 65^{\circ}, 70^{\circ} \leq \theta_{i 2} \leq 100^{\circ}, 70^{\circ} \leq \theta_{i 4} \leq 100^{\circ}$ and $25^{\circ} \leq \theta_{i 5} \leq 65^{\circ}$ for the passive joints. The workspace in Fig. 9 is developed using (16)-(18) with $c_{i 1}=c_{i 2}=0$ and $c_{i 3}=\cos 10^{\circ}$ (for $i=1,2,3$ ) as the nine kinematic constraints. The design can be changed into a translational manipulator by letting $F_{11}=\left[\begin{array}{cccc}1 & 0 & 0 & 75 \\ 0 & 0 & 1 & 0 \\ 0 & -1 & 0 & 0 \\ 0 & 0 & 0 & 1\end{array}\right], F_{12}=\left[\begin{array}{cccc}1 & 0 & 0 & 50 \\ 0 & 0 & 1 & 0 \\ 0 & -1 & 0 & -15 \\ 0 & 0 & 0 & 1\end{array}\right]$ and $c_{i 3}=0$. In this case, $\widehat{\boldsymbol{u}}_{i 1}$ parallel to $\widehat{\boldsymbol{u}}_{i 5}$ and $\widehat{\boldsymbol{u}}_{i 2}$ parallel to $\widehat{\boldsymbol{u}}_{i 4}$ (for $i=1,2,3$ ). Since the position and orientation of the platform can be specified by the $x, y$, $z$ coordinates of the TCP, a two dimensional boundary patch can be developed by solving only one boundary constraint equation. The translational design has larger joint space (with $30 \leq d_{i 3} \leq 50$ ) and workspace, as shown in Fig. 10.

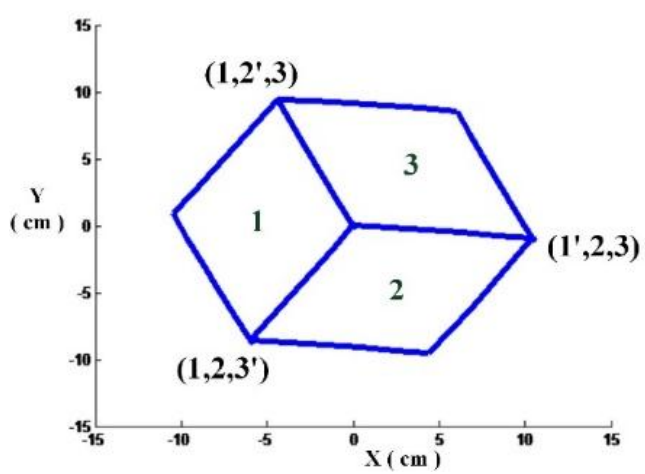

(a) Upper boundary

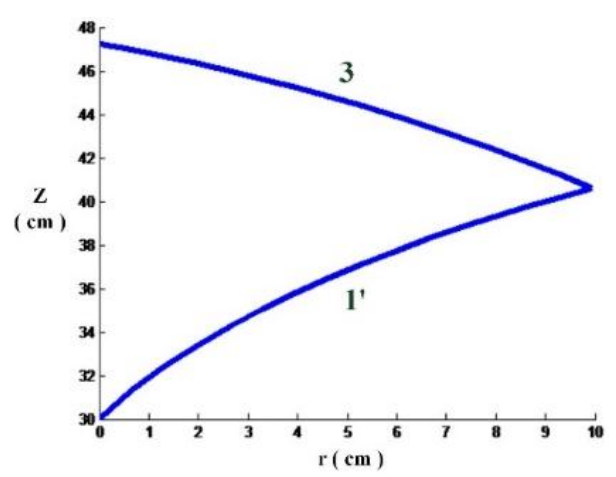

(c) Cross section at $\phi=0^{\circ}$

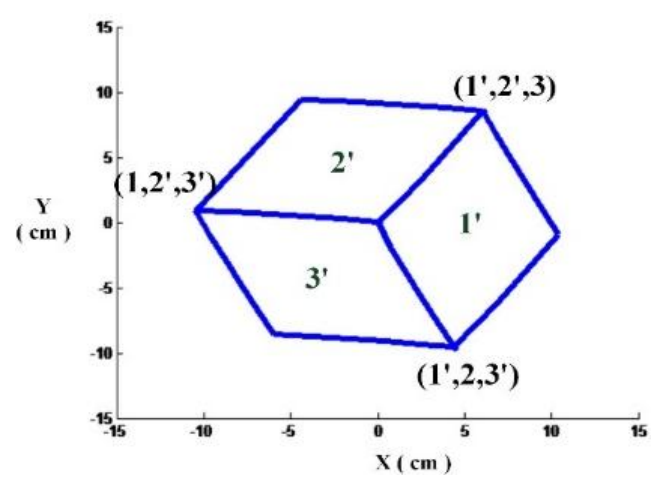

(b) Lower boundary

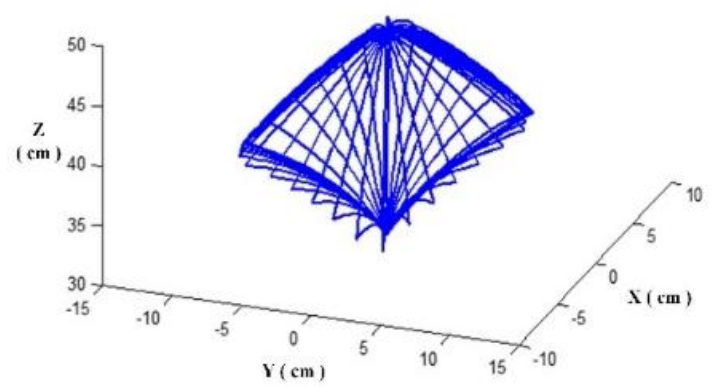

(d) Isometric view

Fig. 9. Workspace of a 3-UPU parallel manipulator.

\subsection{Computation Time}

The numerical examples were programed with Matlab on Intel core 2 Quad CPU with $2.66 \mathrm{GHz}$. Table 2 gives the time needed for the related joint spaces and workspaces. The boundary curves on 24 and 72 cross-sections are developed for the workspace of symmetrical and nonsymmetrical manipulators, respectively. Link interactions rarely occur for 3-limb parallel manipulators, especially when a singularity-free is used to develop the workspace. No link interaction is detected in the search process for all the desired joint spaces. The time required without considering link interactions is given in the parenthesis. It takes much more time for the general 3-UPU manipulator because its direct kinematics 
involves solving twelve nonlinear equations. With the same joint space, it takes $11.74 \mathrm{~s}$ to develop the workspace (in Fig. 8) of the 3-RPS manipulator with smaller spherical joint ranges of $i=1,2,3$.

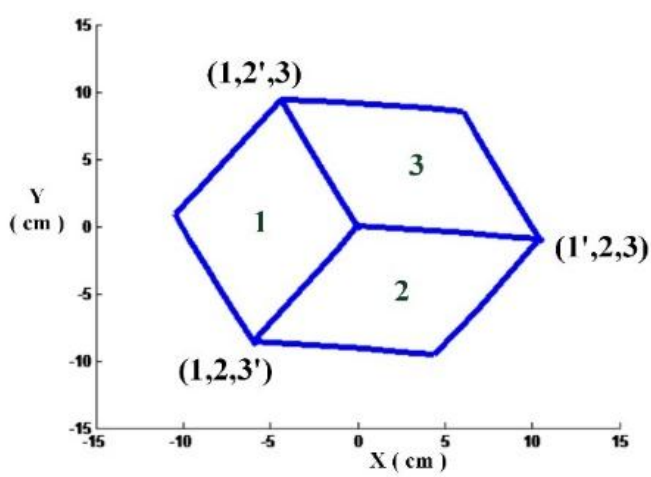

(a) Upper boundary

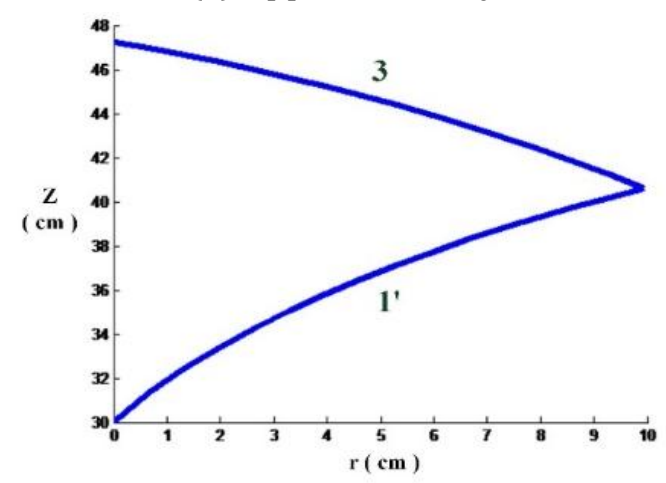

(c) Cross section at $\phi=0$ 。

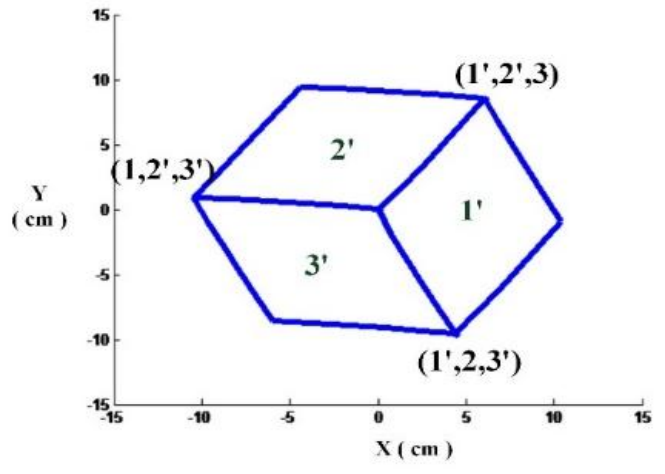

(b) Lower boundary

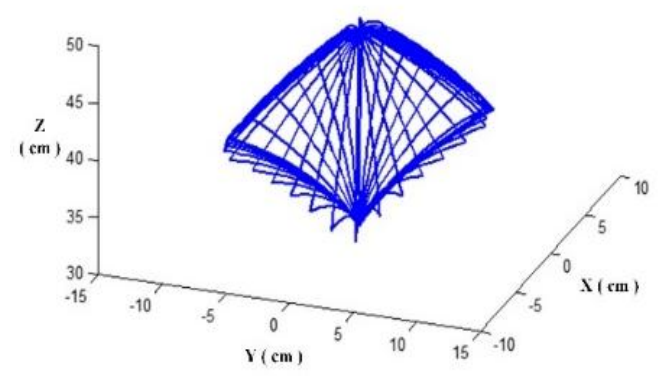

(d) Isometric view

Fig. 10. Workspace of a 3-UPU parallel manipulator.

Table 2. Computation Time for Different Types Manipulator

\begin{tabular}{|c|c|c|}
\hline Type & Joint space & Workspace \\
\hline 3-RPS & $11.47 \mathrm{~s}(10.85 \mathrm{~s})$ & $0.03 \mathrm{~s}$ \\
\hline 3-RRS & $16.90 \mathrm{~s}(15.06 \mathrm{~s})$ & $0.33 \mathrm{~s}$ \\
\hline General 3-UPU & $64.50 \mathrm{~s}(42.5 \mathrm{~s})$ & $4.08 \mathrm{~s}$ \\
\hline Translational 3-UPU & $11.32 \mathrm{~s}(7.32 \mathrm{~s})$ & $0.055 \mathrm{~s}$ \\
\hline
\end{tabular}

\section{Conclusion}

This paper proposes general methods to develop singularity-free and link-interaction-free compatible reachable workspaces for different types of 3-DOF parallel manipulators and studies their characteristics. A workspace boundary usually consists of six patches generated by the six actuator joint limits, and there are two segments for most boundary curves on a cross-section. In general, it needs to solve four equations for a boundary patch of a manipulator with one spherical joint on each limb while the number of equations can significantly increase for a general manipulator without any spherical joints. The effect of passive joint limits, link interactions and singularity are considered in the development process. If necessary, the proposed methods can be employed to develop the related boundary patches for passive joint limits, link interactions or singularity. Link interactions can be neglected because they rarely occur if the workspace is developed from a singularity-free joint space. The equations to develop all the patches can be predicted by solving direct kinematics once and three times, respectively, for a symmetrical manipulator and a general 
manipulator. For a special 3-DOF parallel manipulator (such as a translational manipulator) with the position and orientation of the platform specified by three parameters, a boundary patch can be developed using one equation from a boundary constraint. No kinematic constraint is needed in the development process, unless the boundary constraint contains any joint variables.

\section{Acknowledgment}

Financial support under grant NSC104 - 2221 - E011 - 101 from the National Science Council of Taiwan is gratefully acknowledged.

\section{References}

[1] Li, Y., \& Xu, Q. (2005). Kinematic analysis and design of a new 3-DOF translational parallel manipulator. Journal of Mechanical Design, 128(4), 729-737.

[2] Wang, X., \& Wei, C. (2011). Kinematics and workspace of 3-RUU parallel manipulator. International Conference on Consumer Electronics, Communications and Networks (pp. 438-441).

[3] Huang, M. Z., \& Thebert, J. L. (2010). A study of workspace and singularity characteristics for design of 3-DOF planar parallel robots. The International Journal of Advanced Manufacturing Technology, 51(5-8), 789-797.

[4] Tsai, K. Y., Lo, I.-T., \& Lin, P. J. (2014). Compatible reachable workspaces of symmetrical stewart-gough parallel manipulators. Mechanism and Machine Theory, 77(7), 111-121.

[5] Zenga, R., Daia, S. L., Xieb, W. F., \& Ramab, B. (2015). Constraint conditions determination for singularity-free workspace of central symmetric parallel robots. Proceeding of 15th IFAC Symposium on Information Control Problems in Manufacturing, 48(3), 1930-1935.

[6] Yang, Y. W., \& O'Brien, J. F. (2009). A geometric approach for the design of singularity-free parallel robots. IEEE International Conference on Robotics and Automation, ICRA '09. Kobe.

[7] Tsai, K. Y., \& Lin, J. C. (2006). Determining the compatible orientation workspace of Stewart-Gough parallel manipulators. Mechanism and Machine Theory, 41(10), 1168-1184.

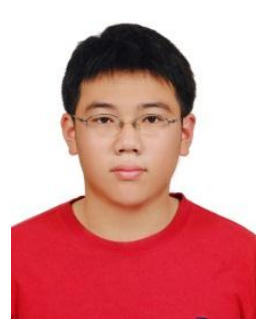

C. K. Huang was born in 1989. He received the M.S. degree in mechanical engineering in 2012 from National Taiwan University of Science and Technology and is currently pursuing the Ph.D. degree from mechanical engineering, National Taiwan University of Science and Technology. His research focuses on kinematics, dynamics, the optimal design and workspace analysis of different types of serial, parallel and redundant manipulators.

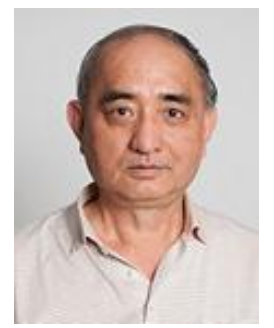

K. Y. Tsai received the B.S. degree in mechanical engineering from the Tatung Institute of Technology, Taipei, Taiwan, in 1975 and his M.S. and Ph.D. degrees from the University of Wisconsin at Milwaukee, in 1986 and 1990, respectively. He is currently a professor in the Department of Mechanical Engineering, National Taiwan University of Science and Technology. His research interests are kinematics, trajectory planning, and the design and analysis of serial, parallel and redundant manipulators. 